\title{
Visualizing Microscopic 3D Displacements of Things over Large Areas
}

\section{Researchers demonstrate a technique that can track displacements as small as $10 \mathrm{~nm}$ with a sensitivity that is independent of field-of-view.}

\author{
By Christopher Crockett
}

( ne way to follow the motion of a particle is to take a series of images of the particle and then track the shifts in its position between image frames. Improving sensitivity in position changes typically requires zooming in, which means there's a tradeoff between the size of step that can be tracked and the area over which the particle can be monitored. Now, Stefano Aime of PSL University in France and colleagues have developed an imaging technique that can visualize 3D displacements of moving elements down to 10 nm-comparable to state-of-the-art microscopy-without sacrificing field-of-view [1]. Dubbed dynamic speckle holography, the technique works with anything that scatters light, unlike other methods that typically require micrometer-sized objects.

To demonstrate their method, the team used it to visualize the deformation of a colloidal gel as a crack propagated. They sandwiched the gel between two glass windows and triggered a

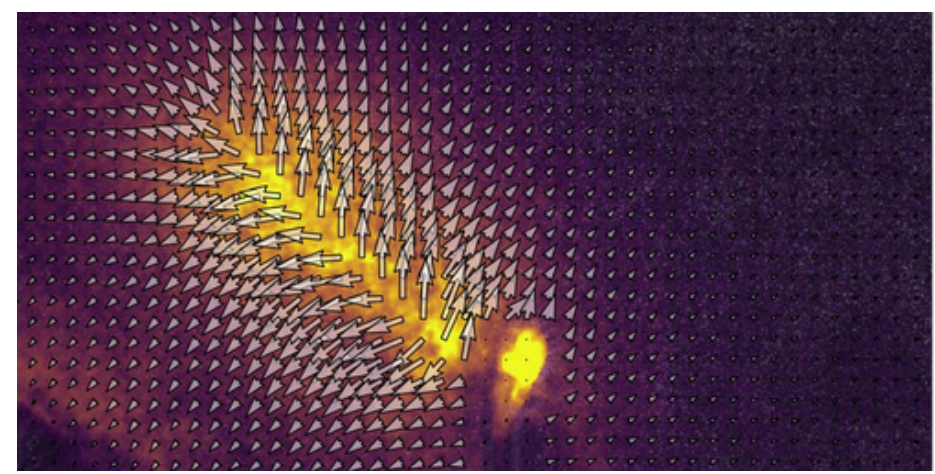

Credit: S. Aime et al. [1] crack by injecting mineral oil into the gel. They then alternately switched on and off two lasers aimed at the gel, with each one illuminating the gel from a different direction. Two cameras, one on each side of the sample, captured the laser light scattered by the gel as the crack grew. Using these "speckle patterns," the researchers showed that they could reconstruct the full 3D deformation of the gel.

This reconstruction shows that the gel deforms in a way that is quite different from predictions based on traditional fracture mechanics-a surprising find, the team says. They add that the result suggests that internal tension in the gel pulls the material apart as the crack propagates.

Christopher Crockett is a freelance writer based in Arlington, Virginia.

\section{REFERENCES}

1. S. Aime et al., "Dynamic speckle holography," Phys. Rev. Lett. 127,088003 (2021). 